\title{
Complicaciones posoperatorias en pacientes con cirugía de válvula mitral
}

\section{Postoperative complications in patients with mitral valve surgery}

\author{
Ángela María Monsalve, Mónica Fernanda Zuluaga-Gómez, \\ Carmen luisa Betancur-Pulgarín, Víctor Daniel Calvo • Pereira (Colombia)
}

\section{Resumen}

Objetivos: determinar las condiciones preoperatorias e intraoperatorias que aumentan el riesgo de morbilidad y mortalidad en el posoperatorio inmediato en los pacientes con cambio de válvula mitral, en la Clínica MAC San Rafael en el periodo del 2014 al 2017.

Material y métodos: se realizó un estudio de casos de tipo cuantitativo, observacional descriptivo, las variables cualitativas se presentaron en frecuencias absolutas, se realizó análisis univariado de los factores predictivos de complicaciones posoperatorias utilizando el riesgo relativo e intervalo de confianza a $95 \%$. Para las variables cuantitativas continuas y discretas se utilizaron medidas de resumen y de tendencia central, como media, mediana, desviación estándar, rango, rango intercuartil.

Resultados: en el presente estudio se reportaron 24 casos de pacientes intervenidos de cambio de válvula mitral, la edad media de los pacientes fue de $58.7 \pm 15.43$ años; no se hallaron diferencias estadísticamente significativas en los promedios de edad entre hombres y mujeres $(p=0.99)$. La hipertensión arterial es una variable estadísticamente significativa $\mathrm{p}=0.03$ IC.17 (1.29-223.13), por cada paciente $<50$ años con hipertensión arterial hay 5.6 pacientes $>50$ años con la misma patología.

Conclusiones: las variables preoperatorias como antecedentes de hipertensión arterial y una clase funcional NYHA III, son comunes en las complicaciones de los pacientes intervenidos sobre la válvula mitral. Además los pacientes que presentaron tiempos de clampeo y cirugía mayor que la media para otros estudios presentaron infección de sitio operatorio y septicemia. (Acta Med Colomb 2019; 44: 91-95).

Palabras clave: complicaciones, válvula mitral, condiciones intraoperatorias.

\begin{abstract}
Objectives: to determine the preoperative and intraoperative conditions that increase the risk of morbidity and mortality in the immediate postoperative period in patients with mitral valve change at the MAC San Rafael Clinic in the period from 2014 to 2017.

Material and methods: a quantitative, observational descriptive case study was conducted, qualitative variables were presented in absolute frequencies, univariate analysis of predictors of postoperative complications was performed using relative risk and $95 \%$ confidence interval. For the continuous and discrete quantitative variables, summary measures and central tendency were used, such as mean, median, standard deviation, range, interquartile range.

Results: in the present study, 24 cases of patients undergoing mitral valve replacement were reported, the mean age of the patients was $58.7 \pm 15.43$ years; no statistically significant differences were found in the average age between men and women $(\mathrm{p}=0.99)$. Hypertension is a statistically significant variable $\mathrm{p}=0.03$ IC .17 (1.29-223.13), for each patient $<50$ years with arterial hypertension there are 5.6 patients $>50$ years with the same pathology.

Conclusions: preoperative variables such as history of arterial hypertension and a functional class NYHA III, are common in the complications of patients operated on the mitral valve. In addition, patients who presented clamping and surgery times greater than the average for other studies presented infection of the operative site and septicemia. (Acta Med Colomb 2019; 44: 91-95).

Keywords: complications, mitral valve, intraoperative conditions.
\end{abstract}

Ángela María Monsalve y Mónica Fernanda Zuluaga-Gómez: Enfermeras. Especialistas en Epidemiología; Carmen Luisa BetancurPulgarín: Magister en Enfermería, Especialista en Epidemiología, Docente Asociada Fundación Universitaria del Área Andina, Investigadora Asociada Colciencias; Dr. Víctor Daniel Calvo: Fundación Universitaria del Área Andina. Pereira (Colombia).

Correspondencia: Ángela María Monsalve, Pereira (Colombia).

E-mail: anmonsalve4@estudiantes.areandina. edu.co

Recibido: 17/IV/2018 Aceptado: 22/II/2019 


\section{Introducción}

En nuestros días es bien conocido que la etiología más frecuente de la estenosis mitral (EM) es la fiebre reumática y se presenta más en mujeres que en hombres; y en la insuficiencia mitral (IM) las causas más habituales son degenerativas o isquémicas, que ocurren de manera más frecuente en países desarrollados y la fiebre reumática en los países en desarrollo, siendo la IM la segunda patología valvular más presentada en Europa. Actualmente para ambos casos son utilizadas las intervenciones quirúrgicas tanto la plastia como la sustitución valvular, aunque se suele elegir la reparación valvular por ser una técnica que conserva el aparato valvular; de igual manera se ha planteado la técnica percutánea, esta por su parte advierte menos complicaciones al no ser una cirugía abierta, debiéndose tener en cuenta un panorama general del contexto de cada paciente (1).

La cardiopatía valvular mitral es objeto de un interés creciente, como también lo es el incremento de patologías valvulares degenerativas a nivel mundial debiéndose a un aumento progresivo de la población mayor. La diferencia clínica según las regiones y los países son variables constantemente, en Cuba por ejemplo las enfermedades del corazón son la primera causa de muerte (2). En Argentina la insuficiencia mitral es la valvulopatía más frecuente y su causa más común es la degenerativa, y dentro de éstas el prolapso valvular es predominante; un estudio realizado en este país mostró en sus resultados, que la sobrevida global fue de $90 \%$ y para aquellos pacientes con afectación de la válvula anterior o bivalvar se determinó como predictor de mortalidad (3).

Múltiples técnicas se utilizan en el mundo en cuanto a la intervención sobre la válvula mitral, pero la reparación de la válvula mitral con el método de Carpentier surgió como el más exitoso. La evolución de las nuevas tecnologías ha conllevado al mejoramiento de procesos y elementos que facilitan la adecuada elección al momento de las intervenciones. No se puede dejar de lado la sustitución de la válvula mitral, técnica a la que se le atribuyen gran número de cirugías realizadas. En un estudio realizado en Cuba el $4.4 \%$ de los pacientes operados con reemplazo valvular mitral fallecieron, los autores resaltan la importancia de evaluar el riesgo quirúrgico preoperatorio, dado que este es un determinante relevante frente a las complicaciones posquirúrgicas (4).

Si bien las ventajas de la reparación valvular en las complicaciones son innegables, se hace necesario el reemplazo para aquellos pacientes con valvulopatía severas, la cual mejora el resultado de estos.

Sin duda hay que prever las posibles complicaciones de la sustitución, ya sea aquellas asociadas a la prótesis, a infecciones de la válvula protésica, a defectos estructurales o a los no estructurales, y debe tenerse en cuenta la principal limitación, para la cual se requieren estudios de las nuevas tecnologías con el fin de mejorar la condición de calcificación y deterioro de estas. Según Schoen y colaboradores, en su estudio realizado en 2016, se practicaban alrededor de
300000 cirugías de la válvula cardiaca, con una mortalidad de 4-9\% en el reemplazo o sustitución de la válvula mitral y los casos en los que en la válvula presentó falla, se requirió la reintervención quirúrgica o generó la muerte entre 60 y $70 \%$ de los pacientes dentro de los 10-15 años posoperatorios (5).

Para el periodo del 2014-2017 en Colombia se evidenció que la primera causa de muerte en hombres y en mujeres fueron las enfermedades del sistema circulatorio, y dentro de estas $49.3 \%$ fueron patologías isquémicas del corazón (6). En el país existe pobre estadística sobre la frecuencia de los cambios valvulares mitrales, sus complicaciones y factores influyentes.

\section{Compromiso bioético}

Basados en la Resolución 008430 de 1993 que genera los lineamientos para la investigación en seres humanos en Colombia, el grupo de trabajadores de la Fundación del Área Andina clasificó esta indagación como una investigación sin riesgo debido a que en el estudio se utilizaron métodos de investigación documentales retrospectivos, como las historias clínicas e informes quirúrgicos, no se realizó ningún tipo de intervención en las variables biológicas, fisiológicas o psicológicas de los individuos, se respetaron los principios éticos para estudio con personas, además del compromiso de hacer parte de la cultura basada en la generación, apropiación y difusión del conocimiento. Este trabajo garantiza los mínimos éticos indicados por el protocolo de Emanuel Wendler: valor social de la investigación, validez científica, selección justa de los sujetos, balance favorable riesgo beneficio.

\section{Material y métodos}

Se realizó un estudio de casos de tipo cuantitativo, observacional descriptivo, sobre las condiciones preoperatorias e intraoperatorias, en el posoperatorio inmediato de cirugía de válvula mitral. La población objeto de estudio está constituida por 24 historias clínicas e informes de los pacientes intervenidos quirúrgicamente de válvula mitral, se revisó la base de datos de la Clínica MAC San Rafael para el periodo comprendido entre el 2014-2017. La información clínica de los pacientes intervenidos de cirugía cardiaca mitral es registrada en la historia clínica y en los informes quirúrgicos, esta información incluye los datos sociodemográficos, los antecedentes familiares de los pacientes, su situación clínica, las variables de la intervención y del posoperatorio inmediato en la UCI, la evolución posterior hasta el alta, así como los datos de los reingresos si se presentan.

\section{Criterios de inclusión}

Historia clínica de pacientes con valvulopatía mitral, intervenidos quirúrgicamente en la clínica MAC San Rafael, en el periodo comprendido entre 2014 y 2017.

\section{Recolección de datos}

Se utilizó como herramienta de tabulación de datos el software Epi-info 7.2. Se creó un instrumento con su respec- 
tivo instructivo para la recolección de los datos de cada una de las variables seleccionadas de acuerdo con la búsqueda bibliográfica y la evidencia científica registrada en ésta y se realizó su respectiva revisión con dos expertos. Este estudio no presenta conflictos de interés.

Para controlar el sesgo de obtención de información al recolectar los datos se hizo control con una doble revisión de las historias clínicas.

\section{Resultados}

Se reportaron 24 casos de pacientes intervenidos de cambio de válvula mitral, la edad media de los pacientes fue de $58.7 \pm 15.43$ años; no se hallaron diferencias estadísticamente significativas en los promedios de edad entre hombres y mujeres $(\mathrm{p}=0.99)$. La razón hombre a mujer del estudio fue 1:1. (Tabla1).

Sólo uno de los pacientes intervenidos tuvo situación crítica preoperatoria, caracterizada por presentar bajo gasto cardiaco y disnea progresiva; ninguno de los pacientes sufrió infartos previos en los últimos 30 días antes del procedimiento quirúrgico; uno de cada cinco pacientes del estudio se le realizó cateterismo cardiaco izquierdo, ninguno de los pacientes presentó endocarditis infecciosa previa a la cirugía y cuatro de cada cinco tuvieron una FEVI (fracción de eyección del ventrículo izquierdo) preservada $>50 \%$.

La hipertensión arterial es una variable estadísticamente significativa $\mathrm{p}=0.03$ IC.17 $(1.29-223.13)$, esta cifra llama la atención dado que la muestra que se obtuvo para el trabajo fue muy pequeña, por cada paciente $<50$ años con hipertensión arterial hay 5.6 pacientes $>50$ años con la misma patología.

En cuanto al sexo y las comorbilidades se tiene que para la hipertensión arterial hay una razón de hombre a mujer de 1:1 que tienen la enfermedad. Existen diferencias estadísticas significativas en la creatinina preoperatoria siendo mayor en los hombres, el $50 \%$ de los hombres tiene valores de $0.94 \mathrm{mg} / \mathrm{dL}$ o menos $0.47 \mathrm{mg} / \mathrm{dL}$ y el otro $50 \%$ valores superiores a éste, no se observaron diferencias estadísticamente significativas entre las variables prequirúrgicas de presiones arteriales, diastólicas y pulmonares entre hombre y mujeres. El $61.1 \%$ de los pacientes mayores de 50 años estaban clasificados con NYHA III (Tabla 2).

En cuanto a las condiciones intraoperatorias por cada válvula mecánica utilizada en una intervención se utilizaron dos biológicas, el promedio de días estancia fue de $17.2 \pm 14.9$ días, el $50 \%$ de los pacientes presentaron tiempos quirúrgicos de 3.77 horas o menos, la diferencia de tiempo aproximado en minutos entre el tiempo de circulación extracorpórea y el tiempo de clamp fue de 10 minutos o menos, el 50\% de los tiempos de circulación extracorpórea fue de 89 min o menos para los hombres y el otro $50 \%$ con tiempos superiores a éste. En las mujeres, el $50 \%$ requirió dos unidades de paquetes globulares o menos, el otro $50 \%$ requirió dos unidades superiores a éste. El total de pacientes reintervenidos por sangrado posoperatorio fueron tres, por cada paciente que requirió
Tabla 1. Distribución absoluta y relativa de los aspectos demográficos de los pacientes de cambio de válvula mitral en una institución de salud de la ciudad de Pereira; 2014-2017.

\begin{tabular}{|l|c|c|c|}
\hline \multicolumn{2}{|c|}{} & $\begin{array}{c}\text { Frecuencia } \\
\text { absoluta }\end{array}$ & $\begin{array}{c}\text { Frecuencia } \\
\text { relativa (\%) }\end{array}$ \\
\hline \multirow{3}{*}{ Sexo } & Mujeres & 12 & 50.0 \\
\cline { 2 - 4 } & Hombres & 12 & 50.0 \\
\hline \multirow{3}{*}{ Grupo de edad } & $>50$ años & 18 & 75.0 \\
\cline { 2 - 4 } & $<=50$ años & 6 & 25.0 \\
\hline \multirow{3}{*}{ Nivel sociodemográfico } & Bajo & 8 & 33.3 \\
\cline { 2 - 4 } & Medio & 14 & 58.3 \\
\cline { 2 - 4 } & Alto & 2 & 8.3 \\
\hline
\end{tabular}

Tabla 2. La distribución de las comorbilidades según el sexo de los pacientes de cambio de válvula mitral en una institución de salud de la ciudad de Pereira 2014-2017.

\begin{tabular}{|c|c|c|c|}
\hline \multicolumn{2}{|l|}{ Comorbilidades } & \multicolumn{2}{|c|}{ Sexo } \\
\hline & & Hombre (\%) & Mujer (\%) \\
\hline \multirow{2}{*}{ Hipertensión arterial } & $\mathrm{Si}$ & $10(83.3)$ & $10(83.3)$ \\
\hline & No & $2(16.7)$ & $2(16.7)$ \\
\hline \multirow{2}{*}{ EPOC } & $\mathrm{Si}$ & $1(8.3)$ & $3(25.0)$ \\
\hline & No & $11(91.7)$ & $9(75.0)$ \\
\hline \multirow{2}{*}{ Insuficiencia renal } & $\mathrm{Si}$ & $1(8.3)$ & $2(16.7)$ \\
\hline & No & $11(91.7)$ & $10(83.3)$ \\
\hline \multirow{2}{*}{ Diabetes mellitus } & $\mathrm{Si}$ & $2(16.7)$ & $1(8.3)$ \\
\hline & No & $10(83.3)$ & 11(91.7) \\
\hline \multirow{2}{*}{$\begin{array}{l}\text { Intervención cardiaca } \\
\text { previa }\end{array}$} & $\mathrm{Si}$ & $4(33.3)$ & $1(8.3)$ \\
\hline & No & $8(66.7)$ & 11(91.7) \\
\hline \multirow{3}{*}{ NYHA } & II & $4(33.3 \%$ & $4(33.3)$ \\
\hline & III & $6(50)$ & $8(6.7)$ \\
\hline & IV & $2(16.7)$ & 0 \\
\hline
\end{tabular}

reintervención por sangrado, siete no la tuvieron. El número de pacientes que requirió marcapasos al salir de la CEC fue 10.

Se presentaron otras complicaciones como neumonía $8.3 \%$, taponamiento cardiaco $8.3 \%$, choque séptico $4.2 \%$, choque hipovolémico $4.2 \%$, cuatro pacientes tuvieron infección del sitio operatorio, la arritmia posoperatoria más notoria fue la fibrilación auricular con una frecuencia de siete casos, un paciente presentó eventos tromboembólicos posterior a fibrilación auricular, a este paciente se le colocó válvula mecánica.

Las características identificadas en los tres pacientes fallecidos fueron las siguientes: el 100\% eran mujeres mayores de 50 años, presentaron enfermedad renal aguda en el posoperatorio, valorada por niveles séricos de creatinina y 
tasa de filtración glomerular a las 72 horas de posoperatorio, además de tener en el preoperatorio clasificación funcional NYHA III. Las variables cualitativas que en el análisis bivariado mostraron significancia estadística $p=0.000$ fueron las complicaciones graves como la insuficiencia renal y la muerte, ya que todos los pacientes con IR posoperatoria fallecieron, además la muerte relacionada con tener diabetes $\mathrm{p}=0.032$ IC 40 (1.74-914.78).

\section{Discusión de los resultados}

A diferencia del estudio de Chao García y colaboradores, donde la clase funcional NYHA que más predomino fue clase NYHA II, en el nuestro más de la mitad de las unidades de análisis eran clase NYHA III, y los antecedentes predominantes fueron la hipertensión arterial, diabetes y EPOC (4).

Según las guías europeas de cardiología uno de los factores de riesgo para eventos tromboembólicos es la fibrilación auricular, que se comprobó en nuestro estudio en el que se presentó un paciente que tuvo evento tromboembólico posterior a una arritmia posoperatoria, la cual fue fibrilación auricular, además se le implantó válvula de tipo mecánica (7). El estudio de Gema Sánchez-Éspin y colaboradores, encontró que en su institución la edad promedio de los intervenidos era de 58.8 años (rango de edad 22-81 años), para este estudio se encontró similitud con una edad promedio de $58.7 \pm 15.43$ años y más de la mitad de las unidades de análisis presentaron una edad superior a 55 años $(8,9)$.

El tiempo de clampeo promedio fue de 72 minutos, lo que supera la media de otros estudios, además los pacientes que presentaron tiempos de clampeo y cirugía mayor que la media para otros estudios tuvo infección de sitio operatorio y septicemia (5).

En relación con los trastornos del ritmo cardiaco en los estudios internacionales (10), mencionan que $13 \%$ de los pacientes que se intervienen sobre la válvula mitral requieren marcapasos para la salida de circulación extracorpórea, para esta investigación lo requirió el $41 \%$ de los pacientes, el ritmo de salida predominante fue ritmo sinusal o nodal. En la revisión documental se menciona la evidencia de bloqueos auriculoventriculares transitorios en el posoperatorio, para este estudio se presentó dos bloqueos auriculoventricular y las demás arritmias como fibrilación auricular revirtieron en un tiempo máximo de seis horas.

Entre las complicaciones posoperatorias más frecuentes según la estadística mundial (11) es aumento en las horas de ventilación mecánica que tienen desenlaces infecciosos como neumonías, aumento en las horas de inotropia y los que se soportaron con inotrópicos por más de 48 horas murieron. Se realizó reintervención quirúrgica por sangrado posoperatorio de los cuales en uno de ellos se presentó infección de sitio operatorio.

Llama la atención que las tres defunciones fueron mujeres, todas con insuficiencia renal posoperatoria, presentaron fibrilación auricular y tenian previamente NYHA III. Se debe tener en cuenta que las variables confusoras no se exploraron en este estudio como causa del tamaño de la muestra.

En el estudio de Julio C Vasquez (4) la principal etiología que requirió remplazo de la válvula mitral en $92.7 \%$ fue a causa de patologías reumáticas, para el presente estudio no se tuvo en cuenta el tipo de enfermedad valvular o etiología.

Entre las limitantes del estudio se presentó el escaso número de los pacientes a pesar de que al inicio del estudio las investigadoras consideraban que el número de unidades de análisis iba a ser mayor.

Otra limitante es la reducción del tema o criterios de inclusión, porque al seguir las recomendaciones de algunos expertos, el tema fue cerrándose hasta quedar centrado en la cirugía de la válvula mitral solamente, lo cual influyó de manera directa en la captación de los pacientes. También se descartaron historias clínicas que no registraban variables de importancia para el estudio, además como criterio de selección se tuvo en cuenta la intervención exclusiva para la válvula mitral por lo que se descartaron 108 unidades de análisis, las cuales incluían revascularización de uno o más vasos.

Logros durante el trabajo, aprendizaje del proceso investigativo, el componente estadístico y la conjugación de los tres pilares fundamentales de los epidemiólogos, epidemiología, investigación y bioestadística.

\section{Conclusiones}

Es difícil hallar significancia estadística en muestras pequeñas; sin embargo, se realizaron análisis bivariados en los que no se encontraron diferencias estadísticamente significativas entre el sexo, edad y nivel socioeconómico.

Las variables preoperatorias como antecedentes de hipertensión arterial y una clase funcional NYHA III, son comunes en las complicaciones de los pacientes intervenidos sobre la válvula mitral.

No se tuvo en cuenta la medicación de los pacientes ni laboratorios aparte de la creatinina que tal vez pudiesen influenciar en las complicaciones intra y posoperatorias, además de la técnica utilizada en cirugía.

El tiempo de clampeo promedio fue de 72 minutos, lo que supera la media de otros estudios, además los pacientes que presentaron tiempos de clampeo y cirugía mayor que la media para otros estudios tuvo infección de sitio operatorio y septicemia.

Los datos arrojados de las variables intraoperatorias cuantitativas no fueron de significancia para las complicaciones posoperatorias en este estudio.

La mortalidad reportada por este estudio tiene en común una ruta de complicaciones posoperatorias como arritmias tipo fibrilación auricular, insuficiencia renal y aumento en las horas de inotropia

\section{Agradecimientos}

Principalmente a Dios, a nuestras familias, nuestros asesores y compañeros. A la Institución por permitirnos realizar el proyecto. $\mathrm{Y}$ en especial a la docencia por su dedicación, comprensión y sobre todo por el aprendizaje que deja en nosotras. 


\section{Referencias}

1. Monzonís AM, Fernández JMV, Assi EA, Raposeiras S. Valvulopatía mitral y tricuspídea Keywords : Medicine (Baltimore) [Internet]. Elsevier; 2017;11(41):2456-64. Available from: http://dx.doi.org/10.1016/S03045412(13)70646-2.

2. Ii G, Chil R, Ii D, I AMM. Morbilidad y mortalidad de la cirugía valvular aórtica. 2012;14-9.

3. Vrancicmtsac JM, Piccininimtsac FF, Camporrotondo M, Espinoza JC, Camou JI, Castro F, et al. Resultados a largo plazo de la plástica mitral en enfermedad degenerativa: prolapso posterior versus anterior o bivalvar. 2014;409-15. Available from: http://www.sac.org.ar/wpcontent/uploads/2014/10/v82n5a10-es. pdf

4. Chao JL, Vázquez CFJ, Mirabal R. Relación de variables preoperatorias y evolución posquirúrgica precoz en pacientes con cirugía de sustitución valvular mitral. 2014;6(1):1-10.

5. Schoen FJ, Gotlieb AI. Heart valve health, disease, replacement, and repair: a 25-year cardiovascular pathology perspective. Cardiovasc Pathol [Internet]. 2016;25(4):341-52. Available from: http://dx.doi.org/10.1016/j.carpath.2016.05.002
6. Hugo V, Castaño A. ( ASIS ) Dirección de Epidemiología y Demografía Bogotá , noviembre de 2016.2016;1-163. Available from: https://www.minsalud.gov.co/ sites/rid/Lists/BibliotecaDigital/ RIDE/VS/ED/PSP/asis-colombia-2016.pdf

7.. Baumgartner H, Falk V, Bax JJ, De Bonis M, Hamm C, Holm PJ, et al. 2017 ESC/EACTS Guidelines for the management of valvular heart disease. Eur Heart J. 2017;38(36):2739-86.

8. Sánchez-Espín G, Otero JJ, Rodríguez EA, Mataró MJ, Melero JM, Porras C, et al. Cirugía valvular mitral mínimamente invasiva. Cir Cardiovasc [Internet]. 2016;23(6):276-81. Available from: http://dx.doi.org/10.1016/j.circv.2016.02.004

9. Fernandez Mesa JE, Patron Garcia KM. Variables asociadas a eventos adversos perioperatorios en pacientes con valvulopatías e hipertensión pulmo- nar. Rev Cuba Cardiol y Cirugía Cardiovasc [Internet]. 2016;22. Available from: http:// www.medigraphic.com/pdfs/cubcar/ccc-2016/ccc163d.pdf\{Russell, 2008 \#5\}

10. Gonzálezl ALF, Lunal D, Ma MA. Bradiarritmias prolongadas después de cirugía de revascularización coronaria. 1995;212-6.

11. Rubiera A, Gonyenechea F, Dedeu A. Revista Cubana de Medicina Intensiva y Emergencias. Hemorragia Intraven en el adulto [Internet]. 2008;16:1020-4. Available from: http://bvs.sld.cu/revistas/mie/vol7_1_08/mie08108.pdf 